\title{
On the Study of Configuration and Kinematics of a 3-DOF Generalized Spherical Parallel Mechanism for Ankle Rehabilitation
}

\author{
jianjun zhang ( $\sim$ zhjjun@hebut.edu.cn ) \\ Hebei University of Technology \\ shuai yang \\ Hebei University of Technology \\ chenglei liu \\ Hebei University of Technology \\ xiaohui wang \\ Hebei University of Technology \\ shijie guo \\ Hebei University of Technology
}

\section{Original Article}

Keywords: Ankle rehabilitation, Parallel mechanism, Kinematic analysis, Parameter optimization

Posted Date: August 17th, 2021

DOI: https://doi.org/10.21203/rs.3.rs-808820/v1

License: (c) (i) This work is licensed under a Creative Commons Attribution 4.0 International License. Read Full License 


\section{Title page}

\section{On the Study of Configuration and Kinematics of a 3-DOF Generalized Spherical Parallel Mechanism for Ankle Rehabilitation}

Jianjun Zhang, born in 1971, is currently a professor and a PhD candidate supervisor at Key Laboratory of Robot Perception and HumanMachine Fusion, Hebei University of Technology, China. He received his $\mathrm{PhD}$ degree from Beijing University of Aeronautics and Astronautics, China, in 2004. His research interests include the robotic mechanics, exoskeleton robot, and rehabilitation robot.

Tel: +86-13820008538; E-mail: zhjjun@hebut.edu.cn

Shuai Yang, born in 1996, is currently a master candidate at Key Laboratory of Robot Perception and Human-Machine Fusion, Hebei University of Technology, China. His research interests include parallel mechanism and rehabilitation robot.

Tel: +86-18165137587; E-mail: yshuai1003@163.com

Chenglei Liu, born in 1995, is currently a PhD candidate at Key Laboratory of Robot Perception and Human-Machine Fusion, Hebei University of Technology, China. His research interests include parallel mechanism and rehabilitation robot.

Tel: +86-15620196305; E-mail: 15620196305@163.com

Xiaohui Wang, born in 1970, is currently an associated professor at Key Laboratory of Robot Perception and Human-Machine Fusion, Hebei University of Technology, China. Her research interests include robotic mechanics.

Tel: +86-13920896167; E-mail: wxhui@hebut.edu.cn

Shijie Guo, born in 1963, is currently a professor and a PhD candidate supervisor at Key Laboratory of Robot Perception and HumanMachine Fusion, Hebei University of Technology, China. He received his PhD degree from Tokyo Institute of Technology, Japan, in 1992. His research interests include the man-machine integration system, nursing robot, and unbound physiological information monitoring system

Tel: +86-15522035068; E-mail: guoshijie@hebut.edu.cn

\section{Corresponding author: Jianjun Zhang E-mail: zhjjun@hebut.edu.cn}




\title{
On the Study of Configuration and Kinematics of a 3-DOF Generalized Spherical Parallel Mechanism for Ankle Rehabilitation
}

\author{
Jianjun Zhang ${ }^{1,2} \cdot$ Shuai Yang $^{1,2} \cdot$ Chenglei Liu $^{1,2} \cdot$ Xiaohui Wang $^{1,2} \cdot$ Shijie Guo $^{1,2}$
}

Received June xx, 201x; revised February xx, 201x; accepted March xx, 201x

(c) Chinese Mechanical Engineering Society and Springer-Verlag Berlin Heidelberg 2017

\begin{abstract}
The kinematic equivalent model of the existing ankle rehabilitation robot is inconsistent with the anatomy structure of the human ankle, which will influence the rehabilitation effect. Therefore, this paper equivalent the human ankle to the UR model and proposes a novel 3-DOF generalized spherical parallel mechanism for ankle rehabilitation. The parallel mechanism has two spherical centers corresponding to the rotation center of the tibiotalar joint and subtalar joint. Via screw theory, the mobility of the parallel mechanism is analyzed, which meets the requirement of the human ankle. Its inverse kinematics is presented and singularities are identified based on the Jacobian matrix. The workspaces of the parallel mechanism are obtained by the search method and compared with the motion range of the human ankle, which shows that the parallel mechanism could meet the motion demand of ankle rehabilitation. In addition, on the basis of the motion/force transmissibility, the performance atlases are plotted in the parameter optimal design space and the optimum region is obtained according to the demands of practical application. The results show that the parallel mechanism can meet the motion requirements of ankle rehabilitation and have excellent kinematic performance in its rehabilitation range, which provides a theoretical basis for the prototype design and experiment verification
\end{abstract}

Keywords: Ankle rehabilitation • Parallel mechanism • Kinematic analysis $\bullet$ Parameter optimization

\section{Introduction}

The human ankle, which is one of the three joints of the human lower extremity, is of fundamental importance for

\footnotetext{
Jianjun Zhang

zhjjun@hebut.edu.cn

1 School of Mechanical Engineering, Hebei University of Technology, Tianjin 300401, China
}

balance, support, and propulsion. Nevertheless, it is particularly susceptible to musculoskeletal and neurological injuries [1]. The traditional treatment of ankle injury highly depends on the one-by-one physical therapy by the doctor, which requires a lot of human resources and heavy labor intensity, and the rehabilitation physician cannot objectively evaluate the rehabilitation status. And evidence suggests that without sufficient rehabilitation: $44 \%$ of people will have future problems and approximately $38 \%$ of people will have recurrent activity limitations affecting their function [2]. However, machine-assisted treatment can collect realtime data during the entire rehabilitation process to make a targeted rehabilitation training for the patient, and it can also ensure the accuracy and repeatability of rehabilitation training.

At present, many researchers have carried out research on ankle rehabilitation robots. Girone [3] developed a 6-DOF ankle rehabilitation robot based on the Stewart platform, but its structure is complex and difficult to control. Dai [4] proposed a 3-UPS/U (U, P and S stand for universal, prismatic and spherical pair, respectively) parallel mechanism for ankle rehabilitation, but the degree of freedom of the mechanism is insufficient. Malosio [5, 6] designed an ankle rehabilitation robot based on the 3-RRR ( $\mathrm{R}$ stands for revolute pair) spherical mechanism. The robot had a remote rotation center, and the ankle center is located at the movement center of the mechanism during the rehabilitation. Li [7-9] presented 3-RRS, 2-UPS/RRR and 3-UPS/RRR parallel mechanisms for ankle rehabilitation. These three mechanisms all equated the ankle with an $\mathrm{S}$ pair,

Hebei Province Key Laboratory of Robot Perception and HumanMachine Fusion, Tianjin 300130, China 
and all had a remote rotation center. Fang [10] proposed a rope-driven parallel mechanism for ankle rehabilitation. The mechanism also equated the ankle to an $\mathrm{S}$ pair, which solves the problem of inertial impact caused by rigid rods. Zhao $[11,12]$ developed a biological fusion ankle rehabilitation mechanism that used the motion characteristics of the ankle to constrain the movement freedom degree of the mechanism. Chen [13] equated the ankle with a spatial RR model and proposed an ankle rehabilitation mechanism based on 3-UPU.

In summary, although the current ankle rehabilitation robot can meet the basic rehabilitation training requirements, it still has certain limitations. Mainly, most ankle rehabilitation robots equate the ankle with an S pair [14] or spatial RR model [15]. The ankle is one of the most complex joints in the human body. Its structure complexity and motion particularity make its axes of motion not intersect at one point, but there are multiple instantaneous rotation axes $[16,17]$. The equivalent of the ankle to the $\mathrm{S}$ pair or spatial RR model will generate human-machine interaction force, which makes the rehabilitation training effect unsatisfactory, and even causes secondary damage to patients. To improve the fitting accuracy of human-machine motion, this paper equates the ankle with the UR model based on the ankle anatomy and motion characteristics and proposes a novel 2UPU/[RR][RRR]/PRPS generalized spherical parallel mechanism for ankle rehabilitation.

The structure of this paper is as follows. In Sect. 2, the UR model is selected with respect to the anatomy structure of the ankle. In Sect. 3, the parallel mechanism based on the UR model is proposed and the structure of the parallel mechanism is performed detailly, and the systematical analysis of the parallel mechanism is carried out, including inverse kinematics, Jacobian matrix, singularity and workspace. In Sect. 4, based on the motion/force transmissibility, the parameters of the parallel mechanism are optimized. The conclusions are drawn in Sect. 5.

\section{The Anatomy Structure and Equivalent Model of the Human Ankle}

The research and analysis of the anatomy structure and motion forms of the ankle is the premise and key to the design of an ankle rehabilitation mechanism, and it is also an important basis to evaluate the safety, man-machine coordinate and comfort of ankle rehabilitation mechanism.

\subsection{The Anatomy Structure of the Human Ankle}

As shown in Fig. 1, the ankle is a highly adaptive multi-axis joint, mainly composed of the tibiotalar joint and the subtalar joint [18]. The tibiotalar joint forms the junction between the distal tibia and fibula of the lower leg and the talus, and the subtalar joint is composed of the talus and calcaneus. Both the tibiotalar joint and the subtalar joint collectively bear the weight and movement of the lower extremity. The main motion forms of the ankle include dorsiflexion/plantarflexion, occurring in the sagittal plane; inversion/eversion, occurring in the transverse plane; adduction/abduction, occurring in the frontal plane [19]. The axes of motion of the ankle are not the fixed axes and have no confluence but changes constantly with the movement of the ankle [18].

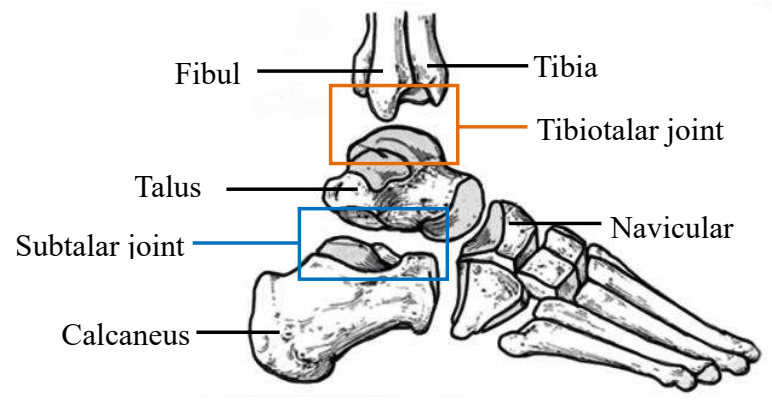

Figure 1 The structure of the ankle

\subsection{Equivalent Model of the Human Ankle}

At present, scholars in the field of ankle rehabilitation generally consider that the equivalent model of the ankle is the spatial RR model or S pair. The two rotation axes of the spatial RR model correspond to the rotation axes of the tibiotalar joint and the subtalar joint of the ankle, but it ignores the motion forms of the ankle. The $\mathrm{S}$ pair considers the motion forms of the ankle and ignores the real structure of the ankle. Zhang [20] proposed a series of ankle equivalent models with respect to the physiological structure and motion characteristics of the ankle. Among them, the UR equivalent model equated the tibiotalar joint with the $\mathrm{U}$ pair and the subtalar joint with the $\mathrm{R}$ pair. The distance from the geometric center of the $\mathrm{U}$ pair to the $\mathrm{R}$ pair was the size of the human talus, as shown in Fig. 2.

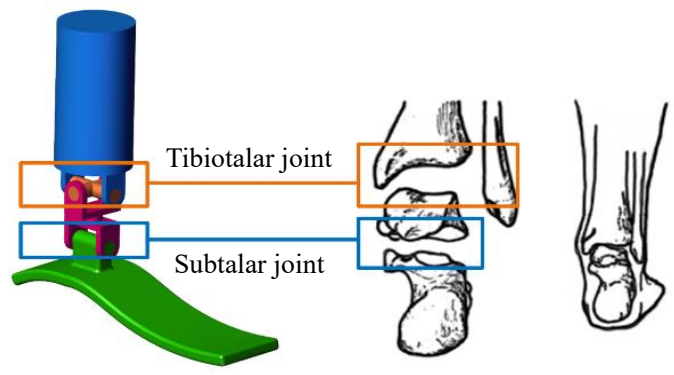

Figure 2 The equivalent model of the human ankle 


\section{The Ankle Rehabilitation Mechanism}

Due to the limitation of the size of the human talus, the serial equivalent model is difficult to meet the rehabilitation requirements. Therefore, a parallel mechanism is designed with respect to the motion forms of the serial equivalent model to improve the rehabilitation accuracy of the ankle.

\subsection{Structure of the Ankle Rehabilitation Mechanism}

Fig. 3 shows the structure of the 2-UPU/[RR][RRR]/PRPS parallel mechanism for ankle rehabilitation based on the UR equivalent model. The parallel mechanism has two rotation centers, namely fixed spherical center $O$ which is equivalent to the rotation center of the tibiotalar joint and moving spherical center $O_{1}$ which is equivalent to the rotation center of the subtalar joint. Point $O$ is located at the intersection of the rotation axes on the fixed platform and point $O_{1}$ is located at the intersection point of the rotation axes on the moving platform. The line between point $O$ and point $O_{1}$ is called the 'double-centered line', and its length is equal to the size of the talus. The parallel mechanism has four limbs between the fixed platform and the moving platform. Limb 1 and limb 2 are the UPU limbs, and the rotation axes connected to the fixed platform are perpendicular to each other and intersect at point $O$. The rotation axes connected to the moving platform intersect at point $O_{1}$. The rotation axes connected to the $\mathrm{P}$ pair are parallel to each other. Limb 3 is the [RR][RRR] limb in which the first two rotation axes intersect at the point $O$ and the remaining rotation axes intersect at the point $O_{1}$. Limb 4 is an unconstraint PRPS limb in which the axis of the $\mathrm{R}$ pair coincides with the direction of the guide rail. The $\mathrm{P}$ pairs of limb 1 and limb 2 and the slider of limb 4 are the actuators.

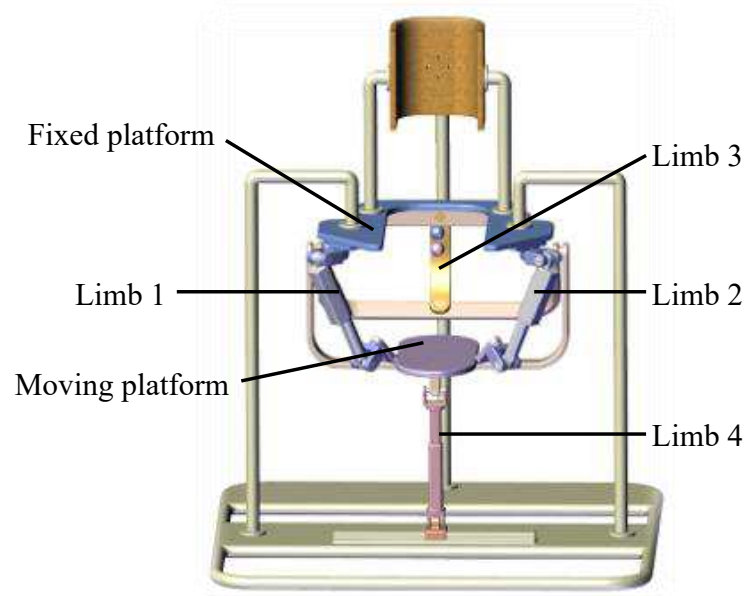

Figure $3 \mathrm{CAD}$ model of the 2-UPU/[RR][RRR]/PRPS parallel mechanism for ankle rehabilitation
As shown in Fig. 4, point $A_{i}(i=1,2)$ is the center of the $\mathrm{U}$ pair connected to the fixed platform in the $i$ th limb. Point $B_{i}$ $(i=1,2)$ is the center of the $\mathrm{U}$ pair connected to the moving platform in the $i$ th limb. Point $A_{3}$ and $A_{3}{ }^{\prime}$ are the centers of the R pairs connected to the fixed platform in limb 3. Point $E_{3}$ is the center of the $\mathrm{R}$ pair connected to the moving platform in limb 3. Point $H$ is the center of the $\mathrm{S}$ pair in limb 4. Point $\mathrm{M}$ is the center of the guide rail.

With point $O$ as the origin of the coordinate system, the $X$-axis coincides with $\mathrm{OA}_{3}$, the $Z$-axis is perpendicular to the fixed platform upward, and the $Y$-axis satisfies the righthand rule, so the fixed coordinate system $O-X Y Z$ is established. Point $O_{1}$ is taken as the origin of coordinates, the $x$-axis is parallel to the direction of $B_{2} B_{1}$, the $z$-axis is perpendicular to the moving platform upward, and the $y$-axis meets the right-hand rule, so the moving coordinate system $O_{1}-x y z$ is established.

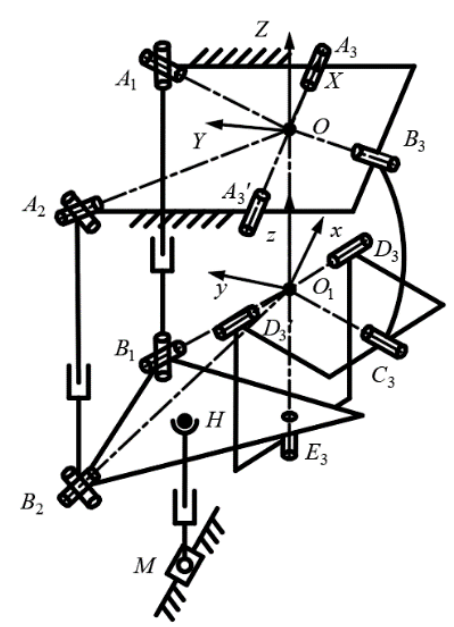

Figure 4 Schematic model of the 2-UPU/[RR][RRR]/PRPS parallel mechanism for ankle rehabilitation

\subsection{Degrees of Freedom}

The DOF of the 2-UPU/[RR][RRR]/PRPS parallel mechanism for ankle rehabilitation is analyzed using screw theory [21]. In screw theory, a unit screw $\$$ is defined as

$$
\$=\left(\begin{array}{ll}
\boldsymbol{s} & \boldsymbol{s}_{0}
\end{array}\right)=\left(\begin{array}{ll}
\boldsymbol{s} & \boldsymbol{r} \times \boldsymbol{s}+h \boldsymbol{s}
\end{array}\right)
$$

where $s$ is a unit vector along the direction of the screw axis, $\boldsymbol{r}$ is the position vector of any point on the screw axis and $h$ is the pith.

The motion screws of limb 1 are given in the fixed coordinate system by 


$$
\left\{\begin{array}{l}
\$_{11}=\left(\begin{array}{ll}
\boldsymbol{s}_{O_{A_{1}}} & \mathbf{0}
\end{array}\right) \\
\boldsymbol{\$}_{12}=\left(\begin{array}{ll}
\boldsymbol{s}_{12} & \boldsymbol{r}_{A_{1}} \times \boldsymbol{s}_{12}
\end{array}\right) \\
\boldsymbol{\$}_{13}=\left(\begin{array}{ll}
\mathbf{0} & \boldsymbol{s}_{A_{1} B_{1}}
\end{array}\right) \\
\boldsymbol{\$}_{14}=\left(\begin{array}{ll}
\boldsymbol{s}_{12} & \boldsymbol{r}_{B_{1}} \times \boldsymbol{S}_{12}
\end{array}\right) \\
\boldsymbol{\$}_{15}=\left(\begin{array}{ll}
\boldsymbol{s}_{O_{1} B_{1}} & \boldsymbol{r}_{O_{1}} \times \boldsymbol{s}_{O_{1} B_{1}}
\end{array}\right)
\end{array}\right.
$$

where $\boldsymbol{s}_{12}$ is a unit vector along the direction of the second rotation axis of the $U$ pair in limb 1 connected to the fixed platform.

Then, employing reciprocal screw theory, the constrain screw of limb 1 can be obtained as

$$
\$_{\mathrm{Cl}}=\left(\begin{array}{ll}
\boldsymbol{s}_{12} & \boldsymbol{r}_{G_{1}} \times \boldsymbol{s}_{12}
\end{array}\right)
$$

where $G_{1}$ is the intersection of the rotation axes in limb 1 connected to the fixed platform and the moving platform.

The motion screws of limb 2 can be expressed as

$$
\left\{\begin{array}{l}
\$_{21}=\left(\begin{array}{ll}
\boldsymbol{s}_{\mathrm{OA}_{2}} & \mathbf{0}
\end{array}\right) \\
\$_{22}=\left(\begin{array}{ll}
\boldsymbol{s}_{22} & \boldsymbol{r}_{A_{2}} \times \boldsymbol{s}_{22}
\end{array}\right) \\
\boldsymbol{\$}_{23}=\left(\begin{array}{ll}
\mathbf{0} & \boldsymbol{s}_{A_{2} B_{2}}
\end{array}\right) \\
\$_{24}=\left(\begin{array}{ll}
\boldsymbol{s}_{22} & \boldsymbol{r}_{B_{2}} \times \boldsymbol{s}_{22}
\end{array}\right) \\
\$_{25}=\left(\begin{array}{ll}
\boldsymbol{s}_{\boldsymbol{O}_{1} B_{2}} & \boldsymbol{r}_{O_{1}} \times \boldsymbol{s}_{O_{1} B_{2}}
\end{array}\right)
\end{array}\right.
$$

where $\boldsymbol{s}_{22}$ is a unit vector along the direction of the second rotation axis of the $U$ pair in limb 2 connected to the fixed platform.

In a similar approach, the constrain screw of limb 2 can be obtained as

$$
\$_{\mathrm{C} 2}=\left(\begin{array}{ll}
\boldsymbol{s}_{22} & \boldsymbol{r}_{G_{2}} \times \boldsymbol{s}_{22}
\end{array}\right)
$$

where $G_{2}$ is the intersection of the rotation axes in limb 2 connected to the fixed platform and the moving platform.

The motion screws of limb 3 can be written as

$$
\left\{\begin{array}{l}
\$_{31}=\left(\begin{array}{ll}
s_{O_{3}} & 0
\end{array}\right) \\
\$_{32}=\left(\begin{array}{ll}
s_{O B_{3}} & 0
\end{array}\right) \\
\$_{33}=\left(\begin{array}{ll}
s_{O_{1} C_{3}} & r_{O_{1}} \times s_{O_{1} C_{3}}
\end{array}\right) \\
\$_{34}=\left(\begin{array}{ll}
s_{O_{1} D_{3}} & r_{O_{1}} \times s_{O_{1} D_{3}}
\end{array}\right) \\
\$_{35}=\left(\begin{array}{ll}
s_{O_{1} E_{3}} & r_{O_{1}} \times s_{O_{1} E_{3}}
\end{array}\right)
\end{array}\right.
$$

The constrain screw of limb 3 can be obtained as

$$
\$_{\mathrm{C} 3}=\left(\begin{array}{ll}
s_{o o_{1}} & \mathbf{0}
\end{array}\right)
$$

Limb 4 is a PRPS limb with six DOF, and there is no constraint screw

According to Eq. (3), Eq. (5) and Eq. (7), the parallel mechanism has three constraint forces. There are arbitrarily distributed in space and are linearly independent, they limit the movement of the moving platform in three directions. So, the moving platform can only rotate in three directions. The DOF of the parallel mechanism can be obtained in terms of the modified G-K formula [22]

$$
F=d(n-g-1)+\sum_{i=1}^{g} f_{i}+v-\zeta=6(13-15-1)+21-0=3(
$$

where $F$ is the number of DOF, $d$ is the rank of the parallel mechanism, $n$ is the number of components, $g$ is the number of joints, $f_{i}$ is the number of DOF for the $i$ th joint, $v$ is the number of redundancy constraints, $\zeta$ is the number of isolated DOF.

\subsection{Inverse Kinematic Analysis}

Let the distance from point $O$ to point $A_{1}$ is $l_{O A_{1}}$. The distance from point $O$ to point $A_{2}$ is $l_{O A_{2}}$. The distance from point $O_{1}$ to point $B_{1}$ is $l_{O_{1} B 1}$. The distance from point $O_{1}$ to point $B_{2}$ is $l_{O_{1 B 2}}$. The length of the 'double-centered line' is $l$. The distance from point $O_{1}$ to point $E_{3}$ is $l_{O_{1} E_{3}}$. The distance from point $E_{3}$ to point $H$ is $l_{E H}$. The angle between the axis of the $\mathrm{U}$ pair connected to the moving platform and the moving platform is $\varphi$. $l_{O A_{1}}=l_{O A_{2}}, l_{O_{1} B_{1}}=l_{O_{1} B_{2}}$.

Euler angles $\alpha, \beta$ and $\gamma$ are used to represent the orientation of the moving platform relative to the fixed platform. The initial attitude of the moving coordinate system $O_{1}-x y z$ is the same as the fixed coordinate system $O$ $X Y Z$. First, $O_{1}-x y z$ is rotated by $\alpha$ about the $X$-axis, then by angle $\beta$ about the $Y$-axis, and finally by angle $\gamma$ about the $Z$ axis. Thus, the rotation transformation matrix can be expressed as

$$
\begin{gathered}
{ }_{o_{1}} \boldsymbol{R}(\alpha, \beta, \gamma)=\boldsymbol{R}(Z, \gamma) \boldsymbol{R}(Y, \beta) \boldsymbol{R}(X, \alpha) \\
=\left[\begin{array}{ccc}
\mathrm{C} \beta \mathrm{C} \gamma & \mathrm{S} \alpha \mathrm{S} \beta \mathrm{C} \gamma-\mathrm{C} \alpha \mathrm{S} \gamma & \mathrm{C} \alpha \mathrm{S} \beta \mathrm{C} \gamma+\mathrm{S} \alpha \mathrm{S} \gamma \\
\mathrm{C} \beta \mathrm{S} \gamma & \mathrm{S} \alpha \mathrm{S} \beta \mathrm{S} \gamma+\mathrm{C} \alpha \mathrm{C} \gamma & \mathrm{C} \alpha \mathrm{S} \beta \mathrm{S} \gamma-\mathrm{S} \alpha \mathrm{C} \gamma \\
-\mathrm{S} \beta & \mathrm{S} \alpha \mathrm{C} \beta & \mathrm{C} \alpha \mathrm{C} \beta
\end{array}\right]
\end{gathered}
$$

where $\mathrm{C}$ represents cosine function, $\mathrm{S}$ represents sine function.

Let the normal vectors of plane $O A_{1} B_{1} O_{1}$ and plane $O A_{2} B_{2} O_{1}$ be $\boldsymbol{n}_{1}$ and $\boldsymbol{n}_{2}$, respectively 


$$
\boldsymbol{n}_{1}:\left\{\begin{array}{l}
\boldsymbol{n}_{1} \cdot O A_{1}=0 \\
\boldsymbol{n}_{1} \cdot \boldsymbol{O}_{1} B_{1}=0
\end{array}, \boldsymbol{n}_{2}:\left\{\begin{array}{l}
\boldsymbol{n}_{2} \cdot O A_{2}=0 \\
\boldsymbol{n}_{2} \cdot \boldsymbol{O}_{1} B_{2}=0
\end{array}\right.\right.
$$

The intersection line of plane $O A_{1} B_{1} O_{1}$ and plane $O A_{2} B_{2} O_{1}$ is the straight line where the 'double-centered line' $\boldsymbol{O} \boldsymbol{O}_{1}$ is located with respect to the structural characteristics of the parallel mechanism, as shown in Fig. 5. Thus, the 'double-centered line' $\boldsymbol{O O}_{1}$ can be obtained as

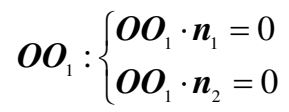

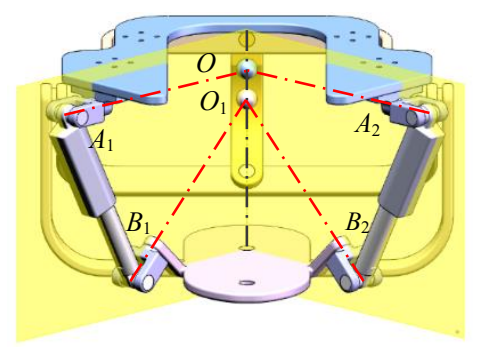

Figure 5 The schematic diagram of the 'doble-centered line'

The position vector of the origin point $O_{1}$ of the moving coordinate system in the fixed coordinate system can be obtained

$$
\boldsymbol{O}_{1}=\left(a_{O_{1}}, b_{O_{1}}, c_{O_{1}}\right)^{\mathrm{T}}
$$

The position vector of the point $B_{1}, B_{2}$ and $H$ in the moving coordinate system can be written as

$$
\left\{\begin{array}{l}
\boldsymbol{b}_{1}=\left(l_{O_{1} B_{1}} \mathrm{C} \varphi \mathrm{C} 45^{\circ}, l_{O_{1} B_{1}} \mathrm{C} \varphi \mathrm{S} 45^{\circ},-l_{O_{1} B_{1}} \mathrm{~S} \varphi\right)^{\mathrm{T}} \\
\boldsymbol{b}_{2}=\left(-l_{O_{1} B_{2}} \mathrm{C} \varphi \mathrm{C} 45^{\circ}, l_{O_{1} B_{2}} \mathrm{C} \varphi \mathrm{C} 45^{\circ},-l_{O_{1} B_{2}} \mathrm{~S} \varphi\right)^{\mathrm{T}} \\
\boldsymbol{h}=\left(0, l_{E H},-l_{O_{1} E_{3}}\right)^{\mathrm{T}}
\end{array}\right.
$$

Through the transformation of Euler angles, the position vector of the points $B_{1}, B_{2}$ and $H$ in the fixed coordinate system can be expressed as

$$
\left\{\begin{array}{l}
\boldsymbol{B}_{1}=\boldsymbol{O} \boldsymbol{O}_{1}+{ }_{o_{1}}^{o} \boldsymbol{R}(\alpha, \beta, \gamma) \boldsymbol{b}_{1} \\
\boldsymbol{B}_{2}=\boldsymbol{O} \boldsymbol{O}_{1}+{ }_{o_{1}}^{o} \boldsymbol{R}(\alpha, \beta, \gamma) \boldsymbol{b}_{2} \\
\boldsymbol{H}=\boldsymbol{O} \boldsymbol{O}_{1}+{ }_{o_{1}}^{o} \boldsymbol{R}(\alpha, \beta, \gamma) \boldsymbol{h}
\end{array}\right.
$$

Then, the lengths of limb 1 and limb $2, l_{1}$ and $l_{2}$ can be obtained as

$$
\left\{\begin{array}{l}
l_{1}=\left|\boldsymbol{A}_{1} \boldsymbol{B}_{1}\right| \\
l_{2}=\left|\boldsymbol{A}_{2} \boldsymbol{B}_{2}\right|
\end{array}\right.
$$

The angle between the vector $\boldsymbol{M H}$ and the guide rail can be expressed as

$$
\theta=\arccos \frac{\boldsymbol{M H} \cdot \boldsymbol{X}}{|\boldsymbol{M H}|}
$$

Then, the slider movement distance $l_{3}$ can be obtained

$$
l_{3}=|\boldsymbol{M H}| \cos \theta
$$

\subsection{Jacobian Matrix}

Jacobian matrix of a parallel mechanism, which represents the mapping between the joint input rates and the moving platform output velocity, is an important tool for analyzing the kinematics performance and singularity of the parallel mechanism. The screw theory is used to establish the complete Jacobian matrix of the parallel mechanism.

The instantaneous screw of the moving platform [23] can

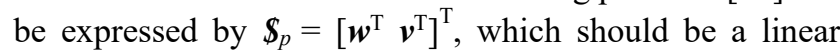
combination of screws in limb $i(i=1 \sim 4)$

$$
\begin{array}{r}
\boldsymbol{\phi}_{p}=\dot{\theta}_{i 1} \boldsymbol{\phi}_{i 1}^{\mathrm{T}}+\dot{\theta}_{i 2} \boldsymbol{\phi}_{i 2}^{\mathrm{T}}+\dot{d}_{i 3} \boldsymbol{\phi}_{i 3}^{\mathrm{T}}+\dot{\theta}_{i 4} \boldsymbol{\phi}_{i 4}^{\mathrm{T}}+\dot{\theta}_{i 5} \boldsymbol{\phi}_{i 5}^{\mathrm{T}}, i=1,2 \\
\boldsymbol{\$}_{p}=\dot{\theta}_{i 1} \boldsymbol{\phi}_{i 1}^{\mathrm{T}}+\dot{\theta}_{i 2} \boldsymbol{\phi}_{i 2}^{\mathrm{T}}+\dot{\theta}_{i 3} \boldsymbol{\phi}_{i 3}^{\mathrm{T}}+\dot{\theta}_{i 4} \boldsymbol{\phi}_{i 4}^{\mathrm{T}}+\dot{\theta}_{i 5} \boldsymbol{\phi}_{i 5}^{\mathrm{T}}, i=3 \\
\boldsymbol{\phi}_{p}=\dot{d}_{i 1} \boldsymbol{\phi}_{i 1}^{\mathrm{T}}+\dot{\theta}_{i 2} \boldsymbol{\phi}_{i 2}^{\mathrm{T}}+\dot{d}_{i 3} \boldsymbol{\phi}_{i 3}^{\mathrm{T}}+\dot{\theta}_{i 4} \boldsymbol{\phi}_{i 4}^{\mathrm{T}}+\dot{\theta}_{i 5} \boldsymbol{\phi}_{5}^{\mathrm{T}}+\dot{\theta}_{i 6} \boldsymbol{\phi}_{i 6}^{\mathrm{T}}, i=4
\end{array}
$$

where $\theta_{i j}$ is the rotational angular velocity of the $j$ th $(j=1 \sim 6)$ joint in the $i$ th limb, $\dot{d}_{i j}$ is the linear velocity of prismatic in the $i$ th limb, and $\$_{i j}$ is the unit screw of the $j$ th in the $i$ th limb.

As mentioned in section 3.2, the parallel mechanism has three constrain screws. The constrain Jacobian matrix can be obtained based on $\$_{\mathrm{C} i} \circ \$_{p}=0$

$$
J_{C}=\left[\begin{array}{cc}
\boldsymbol{r}_{G 1} \times \boldsymbol{s}_{12} & \boldsymbol{s}_{12} \\
\boldsymbol{r}_{\boldsymbol{G} 2} \times \boldsymbol{s}_{22} & \boldsymbol{s}_{22} \\
\mathbf{0} & \boldsymbol{s}_{\boldsymbol{O O} o_{1}}
\end{array}\right]
$$

If we lock the actuated prismatic joint of the $i$ th limb, a new unit transmission screw $\$_{\mathrm{T} i}$ can then be obtained, which represents the intermediate medium that transmits the power from the input to the output terminal [24]. 


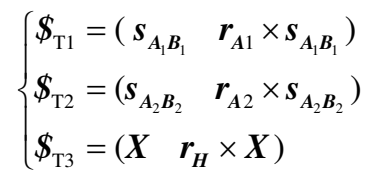

Taking the reciprocity product of both sides of Eq. (18) and Eq. (20) with $\$_{T i}$ yields

$$
\left[\begin{array}{ll}
\boldsymbol{r}_{A 1} \times \boldsymbol{s}_{A_{1} B_{1}} & \boldsymbol{s}_{A_{1} B_{1}} \\
\boldsymbol{r}_{A 2} \times \boldsymbol{s}_{A_{2} B_{2}} & \boldsymbol{s}_{A_{2} B_{2}} \\
\boldsymbol{r}_{H} \times \boldsymbol{X} & \boldsymbol{X}
\end{array}\right] \boldsymbol{\phi}_{p}=\left[\begin{array}{ccc}
\boldsymbol{S}_{\mathrm{T} 1} \circ \boldsymbol{\$}_{13} & 0 & 0 \\
0 & \boldsymbol{\$}_{\mathrm{T} 2} \circ \boldsymbol{\$}_{23} & 0 \\
0 & 0 & \boldsymbol{\$}_{\mathrm{T} 3} \circ \boldsymbol{\$}_{41}
\end{array}\right]\left[\begin{array}{c}
\dot{d}_{13} \\
\dot{d}_{23} \\
\dot{d}_{41}
\end{array}\right]
$$

According to Eq (21) and Eq (23), the velocity model of the 2-UPU/[RR][RRR]/PRPS parallel mechanism can be expressed as

$$
\boldsymbol{J}_{p} \phi_{p}=\boldsymbol{J}_{q} \dot{\boldsymbol{q}}
$$

in which

$J_{p}=\left[\begin{array}{cc}r_{G 1} \times s_{12} & s_{12} \\ r_{G 2} \times s_{22} & s_{22} \\ \mathbf{0} & s_{O O_{1}} \\ r_{A 1} \times s_{A_{1} B_{1}} & s_{A_{1} B_{1}} \\ r_{A 2} \times s_{A_{2} B_{2}} & s_{A_{2} B_{2}} \\ r_{H} \times X & X\end{array}\right]$ denotes the forward Jacobian matrix,

$\boldsymbol{J}_{q}=\operatorname{diag}\left(1,1,1, \boldsymbol{T}_{\mathrm{T} 1} \circ \$_{13}, \$_{\mathrm{T} 2} \circ \$_{23}, \boldsymbol{\$}_{\mathrm{T} 3} \circ \boldsymbol{\$}_{41}\right)$ denotes the inverse Jacobian matrix,

$\dot{\boldsymbol{q}}=\left[0,0,0, \dot{d}_{13}, \dot{d}_{23}, \dot{d}_{41}\right]^{\mathrm{T}}$ denotes the velocity of the input joint.

When the parallel mechanism is away from singularities, we have

$$
\dot{\boldsymbol{q}}=\boldsymbol{J}_{q}^{-1} \boldsymbol{J}_{p} \$_{p}
$$

\subsection{Singularity Analysis}

When the parallel mechanism approaches the singularity configuration, the DOF will be reduced or increased, which makes the parallel mechanism uncontrollable in some directions. According to the determinant of the Jacobian matrix, the singularity configuration of the parallel mechanism is divided into three types [25-26]. The first type, called the inverse singularity, occurs when $\operatorname{det}\left(\boldsymbol{J}_{q}\right)=0$. The second type, called the forward kinematic singularity, occurs when $\operatorname{det}\left(\boldsymbol{J}_{p}\right)=0$. The third type, called the combined singularity, occurs when $\operatorname{det}\left(\boldsymbol{J}_{q}\right)=0$ and $\operatorname{det}\left(\boldsymbol{J}_{p}\right)=0$. Since the human subtalar joint is always below the tibiotalar joint, this paper considers the singularity of the workspace when the moving spherical center is lower than the fixed spherical center.

\subsubsection{Inverse Singularity}

Since the matrix $\boldsymbol{J}_{q}$ is a diagonal matrix, the inverse singularity occurs whenever any of the diagonal elements becomes zero. In addition, all the actuators of the parallel mechanism are prismatic pairs, and the direction of the real unit of the $\$_{\mathrm{T} i}$ is consistent with the direction of the $i$ th actuator. According to the reciprocity product theory, $\boldsymbol{\$}_{\mathrm{T} 1}$ 。 $\$_{13}, \$_{\mathrm{T} 2} \circ \$_{23}$ and $\$_{\mathrm{T} 3} \circ \$_{41}$ are always equal to 1 . So, the parallel mechanism has no inverse singularity.

\subsubsection{Forward kinematic singularity}

The matrix $\boldsymbol{J}_{p}$ consists of three zero-pitch constrain screws and three zero-pitch transmission force screws. A zero-pitch screw can be represented as a line, so the linear dependency among the screws becomes equivalent to the dependency between the lines they represent. Therefore, forward kinematic singularity can be identified by using Grassmann line theory [27] conditions.

The three constrain forces provided by the four limbs to the moving platform are always not in the same plane with the change of the parallel mechanism configuration or the position of the moving platform. According to Grassmann line theory, the rank of the matrix composed of three constrain screws is always equal to 3 . So, the condition for the forward kinematic singularity is that the rank of the matrix $\boldsymbol{J}_{p}$ is 3-5.

(1) the rank of the matrix $\boldsymbol{J}_{p}$ is 3 , there are three possible cases.

Case 1: The union of two flat pencils having a line in common, but lying in distinct planes and with distinct centers. Because the three constrain screws are always not in the same plane, and $\$_{\mathrm{C} 1}$ is perpendicular to $\$_{\mathrm{T} 1}, \$_{\mathrm{C} 2}$ is perpendicular to $\boldsymbol{\$}_{\mathrm{T} 2}$, and the 'doble-centered line' $\boldsymbol{O O}_{1}$ with $\boldsymbol{A}_{1} \boldsymbol{B}_{1}$ and $\boldsymbol{A}_{2} \boldsymbol{B}_{2}$ can never constitute a plane. So, this case does not occur to the parallel mechanism.

Case 2: All lines through a point, but they are not coplanar. This case does not exist because the three transmission force screws are consistent with the direction of the actuators and the three constrain screws do not meet at one point.

Case 3: All lines in a plane, but do not constitute a planar pencil of lines. Since the three constrain screws are always not in the same plane, this case does not exist.

(2) the rank of the matrix $\boldsymbol{J}_{p}$ is 4 , there are three possible cases.

Case 1: All the lines in a plane or passing through one point in that plane. According to the structure of the parallel mechanism, only three transmission force screws may be in the same plane, but the three constrain screws never meet at one point, so this case does not exist. 
Case 2: A one-parameter family of flat pencils, having one line in common and forming a variety. According to the structure of the parallel mechanism, $\$_{\mathrm{C} 1}$ is perpendicular to $\$_{\mathrm{T} 1}$, and is a non-coplanar straight line with $\$_{\mathrm{C} 3}, \$_{\mathrm{T} 2}$ and $\$_{\mathrm{T} 3}$, respectively. In the same way, $\$_{\mathrm{C} 2}$ is perpendicular to $\$_{\mathrm{T} 2}$, and is a non-coplanar straight line with $\$_{\mathrm{C} 3}, \$_{\mathrm{T} 1}$ and $\$_{\mathrm{T} 3}$, respectively. And $\$_{\mathrm{C} 1}$ and $\$_{\mathrm{C} 2}$ can form a plane only at the initial position. At this position, $\$_{\mathrm{T} 1}$ and $\$_{\mathrm{T} 2}$ can form a plane, but $\$_{\mathrm{T} 3}$ and $\$_{\mathrm{C} 3}$ cannot form a plane. So, this case does not exist.

Case 3: All the lines concurrent with two skew lines. According to the analysis of case 2, this case does not exist.

(3) the rank of the matrix $\boldsymbol{J}_{p}$ is 5, there is one possible case: all the lines meeting one line. Since any one of these six lines has at least one set of non-coplanar lines, this case does not exist.

Through the above analysis, the parallel mechanism has no forward kinematic singularity.

\subsubsection{Combined Singularity}

Combined singularity occurs when forward kinematic singularity and inverse singularity occur simultaneously. Because there is no inverse singularity and forward kinematic singularity in the parallel mechanism, the parallel mechanism has no combined singularity.

\section{6 workspace}

Workspace is a necessary condition to measure whether the parallel mechanism can meet the rehabilitation requirements of the human ankle. In this paper, the orientational workspace, moving spherical center and moving platform workspace of the parallel mechanism for ankle rehabilitation are solved. The structural parameters of the parallel mechanism are shown in Table 1.

Table 1. The structural parameters of the mechanism

\begin{tabular}{cc}
\hline Parameters & Number \\
\hline$l_{O A}$ & $275 \mathrm{~mm}$ \\
$l_{O_{1} B}$ & $180 \mathrm{~mm}$ \\
$l$ & $30 \mathrm{~mm}$ \\
$l_{E_{3} H}$ & $120 \mathrm{~mm}$ \\
$\varphi$ & $45^{\circ}$ \\
\hline
\end{tabular}

Under the condition that the moving distances of the $\mathrm{P}$ pairs in limb 1 and limb 2 are limited to $\pm 80 \mathrm{~mm}$ and the rotation ranges of the $U$ pair are $\pm 45^{\circ}$, the workspaces of the parallel mechanism are solved by using the search method with the help of MATLAB. The results are shown in Fig. 6. According to Ref. [28], the motion range of the human ankle and the motion range of the parallel mechanism are listed in Table 2.
Table 2. The motion range of human ankle and the motion range of mechanism

\begin{tabular}{ccc}
\hline Movement Type & $\begin{array}{c}\text { The Motion Range of } \\
\text { Human Ankle/ }{ }^{\circ}[28]\end{array}$ & $\begin{array}{c}\text { The Motion Range of } \\
\text { Parallel Mechanism/ }\end{array}$ \\
\hline Dorsiflexion & $20.3 \sim 29.8$ & 34 \\
Plantarflexion & $37.6 \sim 45.7$ & 51 \\
Inversion & $14.5 \sim 22.0$ & 45 \\
Eversion & $10.0 \sim 17.0$ & 45 \\
Adduction & $22.0 \sim 36.0$ & 76 \\
Abduction & $15.4 \sim 25.9$ & 76 \\
\hline
\end{tabular}

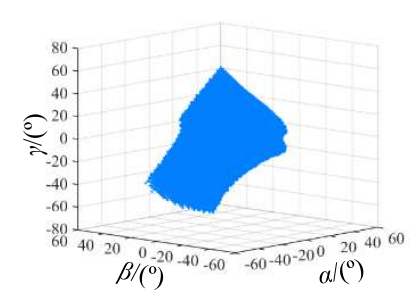

(a) 3D orientational workspace

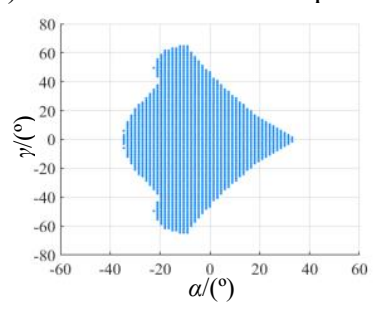

(c) Projection onto $\alpha-\gamma$ plane

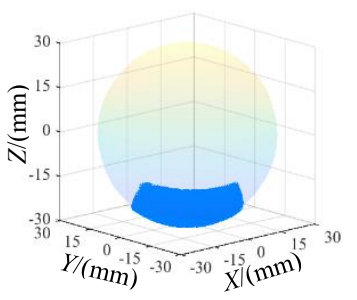

(e) The moving spherical workspace

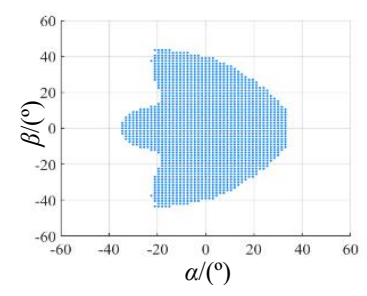

(b) Projection onto $\alpha-\beta$ plane

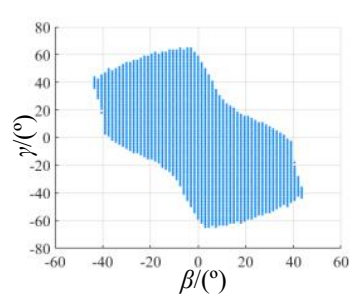

(d) Projection onto $\beta$ - $\gamma$ plane

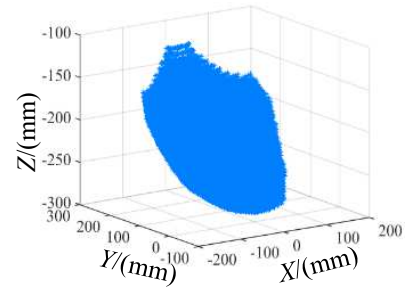

(f) The moving platform workspace
Figure 6 Workspaces of 2-UPU/[RR][RRR]/PRPS

It can be seen from the data in Table 2 that the workspace of the parallel mechanism fully meets the motion range requirements of the human ankle. So, the parallel mechanism for ankle rehabilitation can complete the rehabilitation exercise of the ankle.

\section{Performance Analysis and Optimization}

At present, the performance evaluation indexes applied to parallel mechanisms mainly include the condition number of Jacobian matrix [29], dexterity [30] and motion/force transmissibility [31]. Based on the screw theory, the 
motion/force transmissibility reflects the transmission efficiency of the motion and force of the parallel mechanism from input to output. In this paper, motion/force transmissibility is used as the evaluation standard of the kinematic performance of the ankle rehabilitation parallel mechanism, and the parameters are optimized based on this index.

\subsection{The Motion/Force Transmissibility}

The motion/force transmissibility can be divided into input transmissibility and output transmissibility with respect to transfer objects between the input and output of the parallel mechanism. The input and output transmissibility of a single limb of the parallel mechanism can be expressed as

$$
\begin{aligned}
& \lambda_{i}=\frac{\left|\$_{\mathrm{T} i} \mathrm{o} \$_{\mathrm{I} i}\right|}{\left|\$_{\mathrm{T} i} \mathrm{o} \$_{\mathrm{Ii}}\right|_{\max }} \\
& \eta_{i}=\frac{\left|\$_{\mathrm{T} i} \mathrm{o} \$_{\mathrm{O} i}\right|}{\left|\$_{\mathrm{T} i} \mathrm{o} \$_{\mathrm{O} i}\right|_{\max }}
\end{aligned}
$$

where $\lambda_{i}$ is input transmissibility, $\eta_{i}$ is output transmissibility, $\$_{\mathrm{I} i}$ is the input screw of the $i$ th driving limb, $\boldsymbol{\$}_{\mathrm{T} i}$ is the transmission force screw of the $i$ th driving $\operatorname{limb}, \$_{\mathrm{O} i}$ is the output screw of the $i$ th actuated limb. Both $\lambda_{i}$ and $\eta_{i}$ are within the range of $[0,1]$.

To ensure that each limb has excellent input and output transmissibility, the local transmission index (LTI) of the parallel mechanism is defined as

$$
\Gamma=\min \left\{\lambda_{i}, \eta_{i}\right\}
$$

where $\Gamma$ is a dimensionless index independent of the coordinate system. The closer $\Gamma$ is to 1 , the better the motion/force transmissibility of the parallel mechanism. The closer $\Gamma$ is to 0 , the closer the parallel mechanism is to the singularity.

Furthermore, if we lock any two actuated prismatic joints, and only the actuated prismatic joint of the $i$ th $\operatorname{limb}$ is retained, then only the motion from the $i$ th limb actuated prismatic joint can be transmitted to the moving platform under the action of the $i$ th transmission force. In this situation, the parallel mechanism becomes a single-DOF mechanism, the unit instantaneous screw of the moving platform can be expressed by the unit output screw $\$_{\mathrm{O} i}$. Therefore, according to the reciprocity of the motion screw and the force screw, the unit output screw $\$_{\mathrm{O} i}$ can be obtained

$$
\left\{\begin{array}{l}
\$_{\mathrm{T} j} \mathrm{o} \$_{\mathrm{O} i}=0 \\
\$_{\mathrm{C} j} \mathrm{o} \$_{\mathrm{O} i}=0
\end{array} \quad i \neq j\right.
$$

The LTI of the parallel mechanism can be obtained by putting the output motion screws into Eqs. (26)-(28). The distribution diagrams of LTI of the parallel mechanisms in pure dorsiflexion/plantarflexion $\left(\alpha=0^{\circ}\right)$, inversion/eversion $\left(\beta=0^{\circ}\right)$ and adduction/abduction $\left(\gamma=0^{\circ}\right)$ are drawn, respectively, as shown in Fig. 7. It can be seen from the figures that the LTI of the parallel mechanism in the central area of the workspace is greater than 0.7 , and its value decreases progressively as it leans on the boundary of the workspace. There is no $\Gamma=0$ position in the target workspace, which indicates that the parallel mechanism does not have singularity in the workspace.

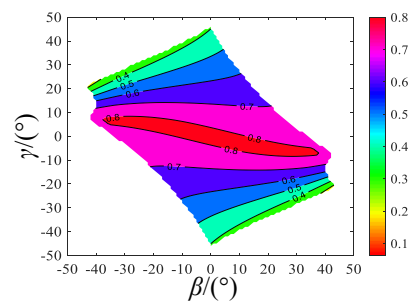

(a) Distribution of LTI when $\alpha=0^{\circ}$

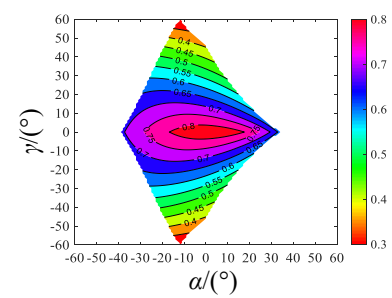

(b) Distribution of LTI when $\beta=0^{\circ}$

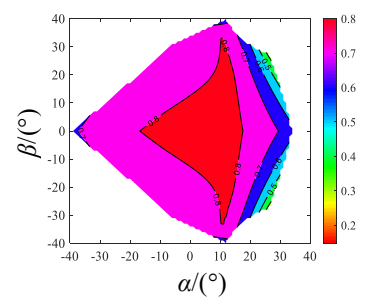

(c) Distribution of LTI when $\gamma=0^{\circ}$
Figure 7 Distribution of LTI for 2-UPU/[RR][RRR]/PRPS

\subsection{Parameter Optimization}

However, LTI can only reflect the motion/force transmissibility of the parallel mechanism in a particular configuration, but not the motion/force transmissibility of the parallel mechanism in the whole workspace. So, the global motion/force transmissibility $\zeta$ is introduced. When $\Gamma \geq 0.7$, the configuration sets of the parallel mechanism are defined as the high-quality transmission workspace. The ratio of the high-quality transmission workspace to the whole workspace is the global motion/force transmissibility $\zeta$ 


$$
\zeta=\frac{\int_{S G} d W}{\int_{S} d W}
$$

where $W$ is the whole workspace, $S G$ is the volume of the high-quality transfer workspace, $S$ is the volume of the whole workspace, $\zeta$ is within the range of $[0,1]$. The closer $\zeta$ is to 1 , the better the kinematic performance of the parallel mechanism.

Substituting the structural parameters in the previous section into Eq. (30), the global motion/force transmissibility of the parallel mechanism is obtained to be 0.33 . It shows that the motion/force transmissibility of the parallel mechanism is not excellent under such structural parameters. So further optimization is necessary.

In this paper, the optimization method proposed in Ref. [32] is used to optimize three structural parameters: the distance from the point $O$ to the point $A_{i}, l_{O A}$; the distance from the point $O_{1}$ to the point $B_{i}, l_{O_{1} B}$; the distance from the point $H$ to the point $E_{3}, l_{E H}$. Firstly, the structural parameters are dimensionless treated as follows

$$
\left\{\begin{array}{l}
D=\frac{l_{O A}+l_{O_{1} B}+l_{E H}}{3} \\
r_{1}=\frac{l_{O A}}{D}, r_{2}=\frac{l_{O_{1} B}}{D}, r_{3}=\frac{l_{E H}}{D}
\end{array}\right.
$$

where $D$ is the normalized factor, $r_{i}$ is the dimensionless parameter of the three optimization parameters.

In consideration of the interference between the various components of the parallel mechanism and the stroke of the push link, the dimensionless parameters shall meet the following conditions

$$
\left\{\begin{array}{l}
r_{1}+r_{2}+r_{3}=3 \\
r_{3} \leq r_{2} \\
r_{2} \leq r_{1}
\end{array}\right.
$$

According to Eq. (32), the parameter optimization region of the parallel mechanism can be obtained, as shown in Fig. $8(\mathrm{a})$. It is projected onto the $s-t$ coordinates to obtain Fig. 8 (b), thereby the three-dimensional space is reduced to the two-dimensional space to reduce the optimization parameters. Its mapping relationship can be obtained as follows

$$
\left\{\begin{array}{l}
r_{1}=-\frac{\sqrt{6}}{6} s+t+\frac{3}{2} \\
r_{2}=-\frac{\sqrt{6}}{6} s-t+\frac{3}{2} \\
r_{3}=\frac{\sqrt{6}}{3} s
\end{array}\right.
$$

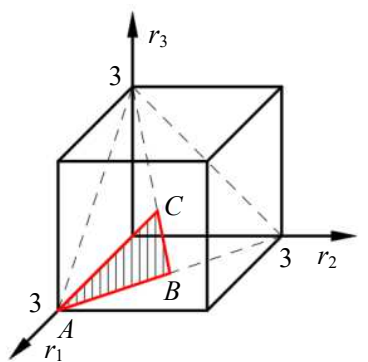

(a) Spatial expression

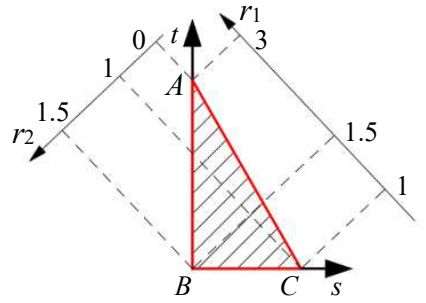

(b)Planar expression
Figure 8 Parameters design space of 2-UPU/[RR][RRR]/PRPS

MATLAB is used to conduct an iterative search on the entire $s$ - $t$ region in Fig. 8(b). The global motion/force transmissibility of the corresponding size is calculated, and the performance atlases are drawn, as shown in Fig. 9. It can be seen from the figure that the global motion/force transmissibility of the parallel mechanism in the range of 0.6 $\leq s \leq 1.22$ and $0 \leq t \leq 0.2$ is greater than 0.5 . It shows that the parameters of the parallel mechanism have an excellent kinematic performance within this range.

Considering the rationality of the layout between the links of the parallel mechanism and the workspace, $s=0.8, t=0.2$, $D=200 \mathrm{~mm}$ are selected. The corresponding mechanism parameters are $l_{O A}=D \cdot r_{1} \approx 255 \mathrm{~mm}, l_{O 1 B}=D \cdot r_{2} \approx 215 \mathrm{~mm}$ and $l_{E H}=D \cdot r_{3} \approx 131 \mathrm{~mm}$. Then the parameters are put into Eqs. (26)-(28), and the distribution diagrams of its LTI are drawn, as shown in Fig. 10.

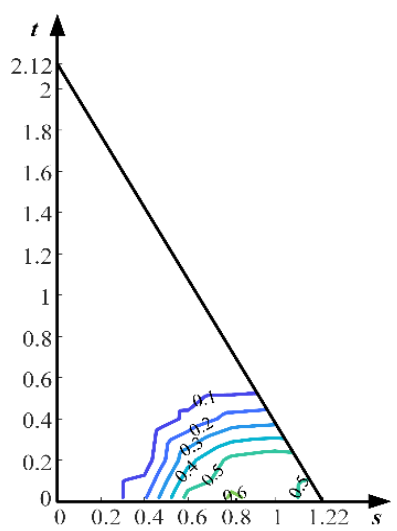

Figure 9 Distribution of LTI in the parameters design 


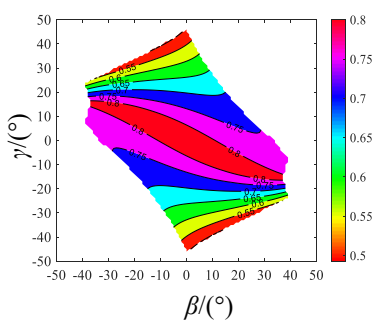

(a) Distribution of LTI of the optimized mechanism when $\alpha=0^{\circ}$

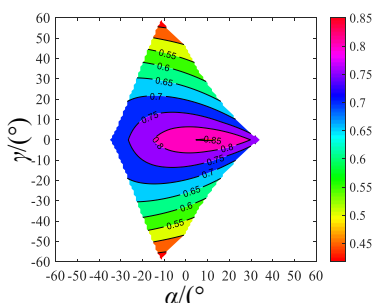

(b) Distribution of LTI of the optimized mechanism when $\beta=0^{\circ}$

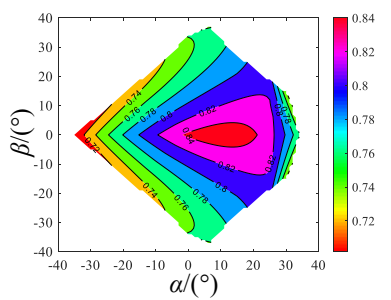

(c) Distribution of LTI of the optimized mechanism when $\gamma=0^{\circ}$
Figure 10 Distribution of LTI for optimized parallel mechanism

By compared the distribution diagrams of LTI of the parallel mechanism before optimization, the area of the high-quality transfer workspace after optimization is significantly increased, and the global motion/force transmissibility of the parallel mechanism after optimization reaches 0.59. It indicates that the kinematic performance of the optimized parallel mechanism has been significantly improved.

\section{Conclusions}

(1) According to the anatomy structure and motion characteristics of the human ankle, the UR equivalent model has been selected and a novel 2UPU/[RR][RRR]/PRPS generalized spherical parallel mechanism for ankle rehabilitation has been presented. The screw theory analysis revealed that the parallel mechanism has three rotational DOFs, which can meet the demand for ankle rehabilitation.

(2) The inverse kinematics of the parallel mechanism was analyzed by the analytical method. According to the screw theory, a complete Jacobian matrix was established, and the singularity was investigated based on inverse singularity, forward kinematic singularity and combined singularity, indicating the parallel mechanism has no singularity. In addition, the workspaces were solved, which shows that the parallel mechanism meets the motion range of the human ankle.
(3) By taking the motion/force transmissibility into consideration, a GTI has been used as the performance evaluation criterion in this paper, and the performance atlases were drawn in the parameter optimal design space. Then, according to the demands of practical application, the optimum region was obtained. The result shows that the ratio of high-quality transmission workspace reached 0.59 , which indicated that the parallel mechanism has excellent kinematic performance in the ankle rehabilitation motion range.

\section{Declaration}

\section{Acknowledgements}

Not applicable.

\section{Funding}

Supported by National Natural Science Foundation of China (Grant No. 52075145), Major Scientific and Technological Achievements Transformation Project of Hebei Province (Grant No. 20281805Z), The Central Government Guides Local Science and Technology Development Funds ((Grant No. 206Z1801G).

\section{Availability of data and materials}

The datasets supporting the conclusions of this article are included within the article.

\section{Authors' contributions}

The author' contributions are as follows: JZ was in charge of the whole trial; SY wrote the manuscript; CL assisted with the process of analysis; XW and SG provided the assistance of theory. All authors read and approved the final manuscript.

\section{Competing interests}

The authors declare no competing financial interests.

\section{Consent for publication \\ Not applicable}

\section{Ethics approval and consent to participate}

Not applicable

\section{References}

[1] M M Zhang, T C Davies, S N Xie. Effectiveness of robot-assisted therapy on ankle rehabilitation-a systematic review. Journal of NeuroEngineering and Rehabilitation, 2013, 10(1): 30.

[2] M J Dong, Y Zhou, J F Li, et al. State of the art in parallel ankle 
rehabilitation robot: a systematic review. Journal of NeuroEngineering and Rehabilitation, 2021, 18(1): 52.

[3] M Girone, G Burzit, M Bouzit, et al. A stewart platform-based system for ankle tele-rehabilitation. Autonomous Robots, 2001, 10(2): 203212.

[4] J A Saglia, N G Tsagarakis, J S Dai, et al. Control strategies for patient-assisted training using the ankle rehabilitation robot (ARBOT). IEEE/ASME Transactions on Mechatronics, 2013, 18(6): 1799-1808.

[5] M Malosio, S P Negri, N Pedrocchi, et al. A spherical parallel three degrees-of-freedom robot for ankle-foot neuro-rehabilitation. 34th Annual International Conference of the IEEE Engineering in Medicine and Biology Society, San Diego, USA, August 28September 1, 2012:3356-3359.

[6] M Malosio, M Caimmi, M Ometto, et al. Ergonomics and kinematic compatibility of PKankle, a fully-parallel spherical robot for anklefoot rehabilitation. 5th IEEE RAS/EMBS International Conference on Biomedical Robotics and Biomechatronics(BioRob), San Diego, USA, August 12-15 2014: 497-503.

[7] D S Li, J F Li, S Wang, et al. 3-RRS Parallel ankle rehabilitation mechanism and motion analysis. Machinery Design and Manufacture, 2015, 8: 4-8. (in Chinese)

[8] J F Li, S C Li, C J Tao, et al. Parallel 2-UPS/RRR ankle rehabilitation mechanism and sports performance analysis. Robot, 2016, 38(2): 144-153. (in Chinese)

[9] J F Li, C H Xu, C J Tao, et al. Performance analysis of 3-UPS/RRR parallel ankle rehabilitation mechanism. Acta Automatica Sinica, 2016, 42(12): 1794-1807. (in Chinese)

[10]R T Yu, Y F Fang, S Guo, et al. Design and kinematic performance analysis of rope driven parallel ankle rehabilitation mechanism. Robot, 2015, 37(1): 53-62. (in Chinese)

[11] H Bian, T S Zhao, X B Tian, et al. Biological fusion rehabilitation institution and its application. Robot, 2010, 32(4): 470-477. (in Chinese)

[12] H Bian, Y H Liu, T S Zhao, et al. Mechanism and kinematics of parallel 2-RRR/UPRR ankle rehabilitation robot. Robot, 2010, 32(1): 6-12. (in Chinese)

[13] Z M Chen, T Yi, H Pan, et al. A 3-DOF parallel ankle rehabilitation mechanism. Journal of Mechanical Engineering, 2020, 56(21): 7078. (in Chinese)

[14] C Z Wang, Y F Fang, S Guo, et al. Design and kinematic analysis of redundantly actuated parallel mechanism for ankle rehabilitation. Robotica, 2015, 33(2): 366-384.

[15] J Dui, G E Johnson. A kinematic model of the human ankle. Journal of Biomedical Engineering, 1985, 7(2): 137-143.

[16] S V S Jan, P Salvia, V Feipet, et al. In vivo registration of both electrogoniometry and medical imaging: development and application on the ankle joint complex. IEEE Transactions on Biomedical Engineering, 2006, 53(4): 759-762

[17] S Canton, W Anderst, M Hogan. In vivo ankle kinematics revealed through biplane radiography: current concepts, recent literature, and future directions. Current reviews in musculoskeletal medicine, 2020, 13(1): 77-85.

[18] B Y Mao, Q J Pang, K R Dai. Artificial ankle surgery. Beijing: People's Military Medical Publishing House, 2015. (in Chinese)

[19] C L Brockett, G J Chapman. Biomechanics of the ankle. Orthopaedics and Trauma, 2016, 30(3): 232-238

[20] C L Liu, J J Zhang, K C Qi, et al. Type synthesis of generalized spherical parallel mechanisms for ankle rehabilitation. Journal of Mechanical Engineering, 2020, 56(19): 79-91. (in Chinese)
[21] S H Li, Z Huang. Comparative analysis of characteristics of the coupled and decoupled parallel mechanisms. Chinese Journal of Mechanical Engineering, 2010, 23(4): 468-476.

[22] Z Huang, J F Liu, Y W Li. On the DOF of mechanism - Searching for the general formula of DOF for 150 years. Beijing: Science Press, 2011. (in Chinese)

[23] W Ye, X X Chai, K T Zhang. Kinematic modeling and optimization of a new reconfigurable parallel mechanism. Mechanism and Machine Theory, 2020, 149: 103850.

[24] M J Song, S Guo, X Y Wang, et al. Dynamic analysis and performance verification of a novel hip prosthetic mechanism. Chinese Journal of Mechanical Engineering, 2020, 33(17): 17

[25] L Kang, S M Oh, W Kim, et al. Design of a new gravity balanced parallel mechanism with Schönflies motion. Proceedings of the Institution of Mechanical Engineers, Part C: Journal of Mechanical Engineering Science, 2015, 230(17): 3111-3134.

[26] S M Kim, B J Yi, W Kim. Forward kinematic singularity avoiding design of a Schönflies motion generator by asymmetric attachment of sunchains. International Journal of Control, Automation and Systems, 2013, 11(1): 116-126.

[27] J P Merlet. Singular configurations of parallel manipulators and Grassmann geometry. The International Journal of Robotics Research, 1989, 8(5): 45-56.

[28] S Siegler, J Chen, C D Schneck. The three-dimensional kinematics and flexibility characteristics of the human ankle and subtalar jointspart I: kinematics. Journal of Biomechanical Engineering, 1988, 110(4): 364-373.

[29] C Gosselin, J Angeles. The optimum kinematic design of a spherical three-degree-of-freedom parallel manipulator. Journal of Mechanical Design, 1989, 111(2): 202-207.

[30] R S Stoughton, T Arai. A modified Stewart platform manipulator with improved dexterity. IEEE Transactions on Robotics and Automation, 1993, 9(2): 166-173.

[31] J S Wang, C Wu, X J Jun. Performance evaluation of parallel manipulators : Motion/force transmissibility and its index. Mechanism and Machine Theory, 2010, 45(10): 1462-1476.

[32] C Wu, X J Liu, L P Wang, J S Wang. Optimal design of spherical 5R parallel manipulators considering the motion/force transmissibility. Journal of Mechanical Design, 2010, 132(3): 031002.

\section{Biographical notes}

Jianjun Zhang, born in 1971, is currently a professor and a $\mathrm{PhD}$ candidate supervisor at Key Laboratory of Robot Perception and Human-Machine Fusion, Hebei University of Technology, China. He received his $\mathrm{PhD}$ degree from Beijing University of Aeronautics and Astronautics, China, in 2004. His research interests include the robotic mechanics, exoskeleton robot, and rehabilitation robot. Tel: +86-13820008538; E-mail: zhjjun@hebut.edu.cn

Shuai Yang, born in 1996, is currently a master candidate at Key Laboratory of Robot Perception and Human-Machine Fusion, Hebei University of Technology, China. His research interests include parallel mechanisms and rehabilitation robot. Tel: +86-18165137587; E-mail: yshuai1003@163.com

Chenglei Liu, born in 1995, is currently a $\mathrm{PhD}$ candidate at Laboratory of Robot Perception and Human-Machine Fusion, Hebei University of Technology, China. His research interests include parallel mechanism and rehabilitation robot. 
Tel: +86-15620196305; E-mail: 15620196305@163.com

Xiaohui Wang, is currently an associated professor at Key Laboratory of Robot Perception and Human-Machine Fusion, Hebei University of Technology, China. Her research interests include robotic mechanics.

Tel: +86-13920896167; E-mail: wxhui@hebut.edu.cn

Shijie Guo, born in 1963, is currently a professor and a $\mathrm{PhD}$ candidate supervisor at Key Laboratory of Robot Perception and Human-Machine Fusion, Hebei University of Technology, China.
He received his $\mathrm{PhD}$ degree from Tokyo Institute of Technology, Japan, in 1992. His research interests include the man-machine integration system, nursing robot, and unbound physiological information monitoring system

Tel: +86-15522035068; E-mail: guoshijie@hebut.edu.cn

\section{Appendix}

Not applicable 